variation in time, reaching a maximum value of about $8 g$. Deceleration and aerodynamic heating, except in the region of the leading edge, are much reduced by employing lift in the descent, although the provision of lifting surfaces entails an increase in weight of the vehicle and the unusual stability and control characteristics pose interesting problems.

The most critical conditions will probably be encountered not in the return from a successfully accomplished orbit, but in emergency re-entry from an abortive ascent. In meeting this contingency there appears to be some advantage in the glider type of vehicle rather than the zero-lift type, but the evidence is by no means conclusive at present.

The question of a successful landing involves many possibilities. An airfield would be a remarkably elusive target in an unpowered glide which, as a result of emergency or mistake, might be initiated over the Pacific Ocean. Probably the glider would have to be regarded as expendable, the occupant being ejected by capsule at a low altitude.

B. E. Beadle

\title{
INTERFACIAL PHENOMENA
}

$\mathrm{T}$ HE biennial conference of the Physical Society, on solid state physics, was held during December 18-20 at the Cavendish Laboratory, Cambridge, under the general title of "Interfacial Phenomena". The conference was attended by about two hundred delegates and twenty-eight papers were presented, which fell into three major groups deseribing, respec tively, various effects associated with films deposited in vacuum, the nature of solid-liquid interfaces and the formation of electrodeposited films, and the structure and properties of internal surfaces. The conference brought together physicists, physical chemists and metallurgists, each with a different approach to the study of interfacial phenomena.

In introducing the papers on films deposited in vacuum, D. W. Pashley (Tube Investments Laboratory, Hinxton Hall) gave first a brief historical survey of the experimental work on the growth of oriented deposits on substrates. He defined the terms 'epitaxy' and 'misfit' and showed that under some conditions epitaxy only occurred with a misfit of less than 15 per cent, but that in many cases misfits of much larger than 15 per cent could be tolerated. The limitations of the various techniques used for the investigation of epitaxy were then outlined, the great power of the electron microscope being emphasized.

The potentialities of the electron microscope in this field of study were fully exploited by G. A. Bassett (Tubo Investments, Hinxton Hall). His experiments on nucleation, orientation and growth of vacuum-deposited metal films on ionic and metallic substrates showed that an oriented layer of gold of about $10 \mathrm{~A}$. mean thickness, deposited on a singlecrystal silver substrate, formed nuclei of 20-200 A. diameter which grew in size rather than number until a continuous film was formed at a mean deposit thickness of about $50 \mathrm{~A}$. When gold was deposited on rock salt a similar effect was observed, but there the gold nuclei formed preferentially at slip and cleavage steps on the rock salt surface.

Reflexion electron diffraction techniques were used by R. C. Newman (Associated Electrical Industries, Aldermaston), A. Glossop and D. W. Pashley (Tube Investments, Hinxton Hall), to study the deposition of copper on silver maintained at different tempera. tures. They found diffraction patterns characteristic of copper with its normal lattice constant when the mean film thickness was $0.8 \mathrm{~A}$. and the substrate temperature $20^{\circ} \mathrm{C}$., but by increasing this temperature to $300^{\circ} \mathrm{C}$. copper patterns were obtained at a mean film thickness of $0.2 \mathrm{~A}$. These results showed that at above a critical mean thickness of much less than one atomic layer, aggregation of the copper occurred on the substrate surface rather than the formation of a uniform film.

Somewhat different results on the influence of imperfections in the substrate upon the mode of epitaxial growth were presented by W. J. Dunning and P. G. Fox (University of Bristol). They examined, with the optical microscope, the growth of ammonium iodide upon mica and observed a uniform epitaxial film spreading outwards until cleavage steps on the mica were reached and then a pyramidal form of growth of the iodide crystals was initiated. The importance of the substrate orientation upon kinetics of film growth was demonstrated clearly by the experiments of J. D. Lawless (University of Virginia), who had oxidized spherical single-crystals of copper in air and in copper sulphate solutions. Differences in the thickness of oxide film were shown by changes in the interference colours from different regions of the spherical surface. Examination by electron diffraction and electron microscopy showed differences in the preferred orientation of the polycrystalline oxide film on various zones of the single crystal.

The epitaxial growth of ice crystals on various substrates and its possible effect upon the formation of rain were discussed by B. J. Mason (Imperial College of Science and Technology, London). $\mathrm{H}_{\Theta}$ described observations on the nucleation of ice on covellite which showed that after nucleation ice crystals may spread laterally on the surface without thickening. The experimental technique involved observing, with the optical microscope, the interference colours produced by the thin crystals on the cleaved covellite surface, and Dr. Mason illustrated his results with colour micrographs of kaleidoscopic variety.

Two further papers on electron diffraction studies of epitaxial growth were presented by M. Blackman (Imperial College of Science and Technology, London), who discussed the epitaxial polymorphism of thallium and crsium halides, and H. Wilman (Imperial College), who gave some new examples of epitaxy. $\mathrm{H}_{\theta}$ showed many diffraction photographs illustrating the different forms of epitaxial growth on ionic, organic and motallic substrates of deposits condensed in vacuo or formed by chemical action.

A group of papers on the properties of surface films were presented next. J. A. Bennet (British Scientific Instrument Research Association) described an apparatus which enabled him to measure the electrical resistivity of a gold film as it was being deposited on either alumina or on a substrate of bismuth oxide on alumina. He found the resistivity decreased as the thickness of the film increased, an effect which he attributed to changes in continuity 
of the film. This conclusion was in agreement with the results given by previous speakers, who showed that in the earliest stages of deposition an epitaxial film was not continuous.

The adhesion of evaporated metal films to glass was discussed by C. Weaver and P. Benjamin (Royal Technical College, Glasgow). The load required to leave a clear scratch through a metal coating deposited in vacuum on a glass substrate was measured and found to depend upon the time which had elapsed after deposition in the case of iron and aluminium, but not for gold. The adhesion of the gold could be attributed to Van der Waals forces, but the rapid increase in the adhesion and its greater value in the case of iron and aluminium could only be accounted for if some chemical bonding occurred between the glass and evaporated layer. Further experiments which tended to confirm that oxygen was necessary to produce this bonding were then described. An entirely different approach to the problems associated with deformation of evaporated layers on substrates was adopted by T. Evans (Tube Investments, Hinxton Hall). An oriented film of gold about $300 \mathrm{~A}$. thick was evaporated on to a single crystal of silver, and on top of the gold film a polycrystalline film of carbon, platinum, iron or chromium was deposited. The composite specimen was then deformed. By dissolving away the silver a specimen thin enough to be examined directly by transmission in the electron microscope remained. This revealed that the presence of the polycrystalline layer modified the slip distribution in the substrate. Dr. Evans concluded that some of the effects could be attributed to adhesion between the polycrystalline and the gold layers.

The first section of the conference was completed by two papers on the surface tension of metallic interfaces. H. Mykura (University of Glasgow) showed that the shape of the grooves produced at grain boundaries when a metal surface is etched thermally depended upon the difference of orientation between the grains, while J. M. Blakely and H. Mykura (University of Glasgow) calculated how an exposed metal surface having a sinusoidal form would change its shape on heating by a process of selfdiffusion leading to mass transport against curvature gradients.

Five papers were presented describing phenornena associated with solid-liquid interfaces. Prof. F. C. Frank (University of Bristol) discussed the kinematics of deposition and dissolution profiles of crystals and showed how the theory of kinematic waves was applicable to this study, while a review of phenomena at metal-electrolyte interfaces was given by $R$ Parsons (University of Bristol), who summarized current ideas about the structure of the electrical double layer between a metal and an electrolyte.

The work of the J. Lucas research group on electrodeposition was introduced by M. J. Pick (University of Birmingham). After reviewing earlier work on electrodeposition he described their new investigations on the deposition of copper on single crystals and polycrystalline cube-texture copper. As in the case of vacuum deposition, the electro-deposited metal initially formed nuclei which eventually grew into each other, forming well-defined crystals. The shape of the nuclei was found to vary with the orientation of the substrate. Further aspects of the work of this research group were then presented by G. C. Storey, T. B. Vaughan and V. R. Howes (University of Birmingham). Changes in surface topography with changes in cathode orientation were determined by detailed light and electron optical examination, and the results obtained were explained in terms of bunching of the growth layers in the manner described in the earlier paper by Prof. Frank. A ciné film of deposits growing in a cell on the stage of an optical microscope were shown; in one sequence growth by a spiral mechanism was elearly recorded.

Further experiments on the growth of electrodeposits of face-centred and body-centred cubic metals on single crystals of face-centred cubic substrates were described by H. Wilman and A. R. N. Reddy (Imperial College of Science and Technology, London). Examination of the deposits by electron diffraction showed that initially epitaxial growth occurred, but that in some cases this was accompanied by rotational disorientation.

The smoothing of electrode surfaces by anodic dissolution or cathodic deposition was discussed by J. Edwards (British Non-Ferrous Metals Researeh Association), who showed how the potential distribution, hence the rate of solution or deposition, was influenced by the topography of the electrode surface.

The papers on internal surfaces were introduced by J. Nutting (University of Cambridge). He outlined first the forms of internal surfaces thought to exist in pure metals, single-phase and two-phase alloys, and then discussed in more detail the structure of interphase interfaces. New results, obtained by transmission electron microscopy of thin metal foils, on the coherent interfaces found in aluminium alloys after suitable aging treatments were described, and the transition from coherent to partially coherent interfaces was shown to occur by the introduction of suitable structural dislocations.

The properties of grain boundaries are frequently discussed, but not often measured. An attempt to obtain quantitative information was described by G. M. Leak (British Iron and Steel Research Association, Sheffield), who showed how the dependence of temperature of the internal friction of iron could be related to its grain size. The relaxation time of the grain boundary damping was also found to be dependent upon alloy content, different results being obtained with interstitial and substitutional elements. P. B. Hirsch and M. J. Whelan (University of Cambridge) and R. Bullough (Associated Electrical Industries, Aldermaston) were more concerned with low-energy interfaces associated with deformation twins. Drs. Hirseh and Whelan described a mechanism for the formation of deformation twins and then discussed this in relation to the contrast effects obtained when examining thin metal foils of stainless steel in the electron microscope, while Dr. Bullough presented a theoretical treatment of the properties of structural dislocations and their movement during the extension of a twin interface.

A series of papers on the influence of interphase interfaces upon the mechanical properties of alloys were then presented. Experiments on the plastic deformation of aged aluminium-copper and aluminium-silver alloys were described by A. Kelly (University of Cambridge), who showed that after aging an aluminium-copper alloy to produce G.P. [I] zones the critical resolved shear stress at a given elongation was very sensitive to temperature, whereas after aging to produce G.P. [2] zones, or the spherical zones in aluminium-silver alloys, there was no thermally activated component. $\mathrm{H}_{\Theta}$ deduced from these results that, with the aid of thermal fluctuations, dislocations could be made to pass through the narrow 
G.P. [1] zones, but with the larger and thicker G.P. [2] zones thermal activation could never provide sufficient energy for the disruption of the zones. The mechanism of hardening in aluminium-copper alloys was discussed further by D. Dew-Hughes and W. D. Robertson (Yale University). They earried out tensile tests on single crystals of alloys which contained between 1 and 5 per cent copper and which had been aged at different temperatures and then used the results obtained to test the predictions of Orowan, Fisher Hart and Pry on the relationships between the shear stress and the particle spacing. The conclusion was reached that a modification of the Fisher Hart and Pry relationship gave agreement between the observed and ealculated results. To account fully for the effects of aging upon the critical resolved shear stress, Dew-Hughes and Robertson derived the stresses required to force dislocations through the solid solution, then to pull them through the coherent strain fields surrounding the zones and finally to disrupt the zones. The sum of these three component stresses agreed closely with the observed shear stresses.

The conclusions of Kelly and of Dew-Hughes and Robertson on the modes of dislocation precipitate interactions were supported by the results presented by G. Thomas and R. B. Nicholson (University of Cambridge). They examined in the electron microscope thin foils of suitably aged aluminium alloys and observed how the dislocations interacted with coherent, partially coherent and incoherent precipitates. Clear evidence for the passage of dislocations through coherent and partially coherent precipitates was presented. But there was little evidence for the dislocation loops forming around precipitate particles as predicted by Fisher Hart and Pry. Further results on precipitate - dislocation interaction were given by E. Smith (Associated Electrical Industries, Aldermaston), who deseribed the effects of hydride precipitates upon the plastic deformation of titanium and showed how the reversible twinning found in pure titanium was hindered by the hydride precipitates. Under conditions of rapid loading cracks developed from the interface between the hydride and the hindered twin.

The generation of dislocations at incoherent interfaces was discussed by J. W. Mitchell and A. S. Parasnis (University of Bristol). They showed how, in a dispersion of glass spheres in silver chloride, the stresses developed at interfaces due to differences in thermal contraction of the two phases could lead to the formation of dislocations by a process of prismatic punching. Optical micrographs were shown of the dislocation loops and their spatial distribution within the silver chloride matrix. Another function of interfaces, as a source of vacancies, was demonstrated by R. S. Barnes (Atomic Energy Research Establishment, Harwell) and Prof. A. H. Cottrell (University of Cambridge), who had investigated the effects associated with the annealing of radiation damage in copper produced by bombardment with $\alpha$-particles. They found small bubbles were produced on anneal. ing and reasoned that these were formed by the diffusion and aggregation of vacancies from either the nearest free surface or internal surfaces such as large-angle grain boundaries; convincing proof that twin interfaces did not act as sources for vacancies was produced. The influence of curvature on the energy of incoherent interfaces was illustrated by a film presented by G. Thomas and M. J. Whelan (University of Cambridge). Thin foils of an aluminium -4 per cent copper alloy were aged at temperatures between $350^{\circ}$ and $450^{\circ} \mathrm{C}$. on a heating stage in the electron microscope while the structural changes as observed on the fluorescent screen of the microscope were recorded with a cine camera. The nucleation and growth of the $\theta$ phase was elearly revealed together with the subsequent growth of the larger particles at the expense of the smaller ones.

From the conference it appeared that at the present stage of development the physicists and metallurgists had more to offer than the physical chemists towards our understanding of interfacial phenomena. The advances made since the introduction of electron diffraction tecluniques for the study of surfaces have been very great, but with the advent of the electron microscope into this field of study a much more detailed investigation of surface morphology has become possible. The explanation of why diffraction patterns could be obtained from deposits of less than one atom mean thickness has now been found, but it is still difficult to account for the preferential formation of epitaxial nuclei at lattice defects. Perhaps it is here that the physical chemists can be of help.

\section{THE COLOMBO PLAN}

\section{ANNUAL REPORT}

\begin{abstract}
$\mathrm{T}$ HE seventh annual report of the Consultative Committee for the Colombo Plan for Co-operative Economic Development in. South and South-East Asia, which by agreement at the Singapore meeting of the Committee in October 1955 has been extended from July 1957 to June 30,1961 , is the last to appear before the future of the Plan is again considered at its 1959 meeting in Indonesia. The present report*, which covers the year 1957-58, records continuing increase in industrial production in the area as a whole, but in India the rate of expansion dropped from 8 per cent in $1956-57$ to 1.5 per cent, which, however, accounted for most of the 13 per cent increase in output of electric power. Since 1950

- The Colombo Plan for Co-operative Economic Development in South and South-East Asia-Seventh Annual Report of the Con(Cmnd. 610.) (London: H.M. Stationery Office, 1958.) 8s. net.
\end{abstract}

training has been given to more than 15,500 trainees from countries in the area, and the services of nearly 8,000 experts provided by members of the Colombo Plan and by agencies of the United Nations. During 1957 expenditure on technical assistance under the Colombo Plan Technical Co-operation Scheme in. creased to about $£ 3$ million, reaching a total of $£ 9.78$ million since 1950 , and during the first six months of 1958 it reached 3.9 million dollars.

Almost half of this expenditure has been for the provision of study and training courses, about a quarter for the supply of experts and the remainder on technical equipment. By the end of June 1958 expenditure on technical equipment reached a total of $£ 2.35$ million, and the number of scholarships and training awards granted by member countries totalled 6,952 , an increase of 1,330 on the previous year. 